\title{
Effect of ginsenoside-Rg1 on experimental Parkinson's disease: A systematic review and meta-analysis of animal studies
}

\author{
YI-BO HE ${ }^{1}$, YONG-LIN LIU ${ }^{2}$, ZHENG-DONG YANG ${ }^{1}$, JIA-HONG LU ${ }^{3}$, \\ YAO SONG ${ }^{4}$, YAN-MING GUAN ${ }^{1}$ and YI-MIN CHEN ${ }^{1}$ \\ ${ }^{1}$ Department of Clinical Laboratory, The First Affiliated Hospital of Zhejiang Chinese Medical University, Hangzhou, \\ Zhejiang 310006; ${ }^{2}$ Reproductive Center, Sanya Maternal and Child Health Center, Sanya, Hainan 572000; \\ ${ }^{3}$ Department of Obstetrics and Gynecology, The First People's Hospital of Xiaoshan, \\ Hangzhou, Zhejiang 311200; ${ }^{4}$ Department of Acupuncture, The Second Affiliated Hospital of \\ Zhejiang Chinese Medical University, Hangzhou, Zhejiang 310015, P.R. China
}

Received March 3, 2020; Accepted February 2, 2021

DOI: $10.3892 /$ etm.2021.9984

\begin{abstract}
Previous studies have reported that ginsenoside-Rg1 (G-Rg1) was able to mitigate the loss of dopaminergic neurons in animal models of Parkinson's disease (PD). The present study provided a systematic review and meta-analysis of preclinical studies to pool current evidence on the effect of G-Rg1 on neurogenesis in the treatment of PD. Eligible studies were identified through a search from six databases: PubMed, EMBASE, Web of Science, VIP, Chinese National Knowledge Infrastructure and the Wanfang database. Primary outcomes were tyrosine hydroxylase (TH)-positive cells in the nigra, Nissl staining-positive cells in the nigra, pole test time and dopamine (DA) levels in the striatum. A total of 18 eligible studies were identified, involving 343 animals. Of these, 13 reported a significant relationship between G-Rg1 and improved TH-positive cells in the nigra compared with the control group $(\mathrm{P}<0.00001)$. Furthermore, 3 studies reported a significant relationship between G-Rg1 and improved Nissl-positive cells in the nigra compared with the control group $(\mathrm{P}<0.00001)$. In addition, 4 studies reported a significant effect of G-Rg1 to reduce the total pole test time compared with that in the control group $(\mathrm{P}=0.001)$. A total of 3 studies indicated a significant association between G-Rg1 and improved DA levels in the striatum compared with the control group $(\mathrm{P}<0.00001)$. These results suggested that G-Rg1 has positive effects in attenuating damage in models of $\mathrm{PD}$, and thus, it is a potential candidate neuroprotective drug for human PD.
\end{abstract}

Correspondence to: Mr. Yi-Min Chen, Department of Clinical Laboratory, The First Affiliated Hospital of Zhejiang Chinese Medical University, 54 Youdian Road, Hangzhou, Zhejiang 310006, P.R. China

E-mail: cym3338@163.com

Key words: ginsenoside-Rg1, Parkinson's disease, animal model, meta-analysis

\section{Introduction}

Parkinson's disease (PD) is the second most prevalent neurodegenerative disorder after Alzheimer's disease (1). PD is characterized by progressive loss of nigral dopamine neurons and decreased dopamine levels in the striatum of the basal ganglia. Patients with PD present with symptoms such as tremor at rest, rigidity, bradykinesia, postural abnormalities and the freezing phenomenon (1). Studies have reported a prevalence of PD of 0.5-1\% among individuals aged 65-69 years and $1-3 \%$ among those aged 80 years and above (2). Despite nearly 50 years of research, no effective treatment has been developed for PD (3). L-DOPA has been the most widely used PD treatment, however, its therapeutic effects decrease with long-term therapy. Furthermore, numerous alternative therapies produce severe side effects during therapy (4). The current pharmacological treatments for PD only treat symptoms and cannot stop the progressive loss of dopaminergic neurons in patients with PD (5). Therefore, it is essential to discover other potential therapeutic agents with better efficacy for PD. Furthermore, reports of the failures of candidate drugs for PD suggest the need for strategies to enhance the probability of effective translation into animal research, therefore providing improved clinical benefits (6). Numerous preclinical systematic reviews have been proposed to promote candidate drug development and discovery as well as clinical drug development. For centuries, ginseng has been used in Traditional Chinese Medicine as a tonic for vitality and stamina. The major active components of ginseng are ginsenosides (7), which exert beneficial effects in humans, including alleviating learning and memory impairment as well as reversing pathological and physiological changes induced by stress and aging. Ginsenoside-Rg1 (G-Rg1) is the most significant bioactive component responsible for the pharmaceutical actions of ginseng (8). It has a wide range of neurotrophic and neuroprotective effects and low toxicity (9). In vivo studies have reported that G-Rg1 protects dopaminergic neurons against glutamate, 1-methyl-4-phenyl-1,2,3,6-tetrahydropyridine (MPTP) and rotenone toxicities (10-12). Chen et al (13) demonstrated 
the protective effect of Rg1 against MPTP-induced nigral neuronal loss. Heng et al (14) reported that Rg1 improved animal survival rates, dopamine loss, motor neuron deficits and abnormal induced ultrastructural changes.

However, to date, only a small number of systematic reviews have established the effects of G-Rg1 in animal models of PD. Song et al (15) published a systematic review using tyrosine hydroxylase (TH)-positive cells in the nigra as the outcome, which is insufficient to judge dopamine neuron loss (16). Therefore, in the present study, further outcomes were included in a meta-analysis, including the number of Nissl stain-positive cells. The majority of the published experimental studies have small sample sizes. Systematically reviewing and meta-analyzing all of these studies in an objective manner is likely to offer reliable and credible evidence on whether a G-Rg1 therapy is effective in experimental PD, allowing for the selection of optimal drug administration requirements for clinical trials. Therefore, a systematic review and meta-analysis was performed to provide evidence supporting the role of G-Rg1 as a neuroprotectant in experimental PD. TH-positive cells, pole test times, Nissl-positive cell counts and DA levels were integrated to perform the meta-analysis.

\section{Materials and methods}

Search strategy. The search strategy was designed according to the criteria of the Preferred Reporting Items for Systematic reviews and Meta-Analyses statement and with no language restrictions (17). An independent search of studies on the effects of G-Rg1 therapy on PD was performed in the following databases from their inception to 2019: PubMed, EMBASE, Web of Science, VIP, Chinese National Knowledge Infrastructure and Wanfang databases. References of articles and reviews of interest were also scanned for additional relevant studies.

The literature search for the meta-analysis was restricted to published animal studies. In addition, references of relevant original papers and review articles were screened. Using the grouped terms, the PubMed search strategy was as follows and was altered to suit other databases: i) 'Paralysis agitans'; ii) 'idiopathic Parkinson's disease'; iii) 'Parkinsons disease'; iv) 'Parkinson's disease'; v) 'Parkinson disease'; vi) 'Parkinsonism'; vii) or/i-v; viii) 'ginseng ginsenoside'; ix) 'ginsenoside-Rg1'; x) 'G-Rg1'; xi) 'Rg1'; xii) or/vii-xi, vi and xii.

Selection criteria. The included studies assessed the effects of G-Rg1 in animal models of PD, with the outcomes measured being TH-positive cells in the nigra, Nissl-positive cells in the nigra, and pole test time and/or dopamine (DA) level in the striatum. The following inclusion criteria were established: i) Studies testing the effect of G-Rg1 in animal models of $\mathrm{PD}$; ii) in the treatment group, the TH-positive cells, pole test times, and/or DA levels were compared with vehicle-treated or untreated model animals; and iii) in the treatment group, G-Rg1 was not tested in combination with other neuroprotective agents. The pre-specified exclusion criteria were as follows: i) Reviews, case reports, abstracts, letters or comments, as well as clinical trials; ii) studies not measuring TH-positive cells and/or pole test times and/or Nissl-positive cell counts and/or DA levels as the outcome; and iii) studies not reporting the effect of G-Rg1 in PD. TH-positive cell counts and DA contents are commonly used to measure dopaminergic neurons in the nigra and the striatum, respectively, in animal models of PD $(18,19)$. The pole test is an effective method of estimating bradykinesia and motor coordination in animal models (20). The PD models used have yet to predict the efficacy of a single effective treatment, although they have been useful in selecting certain symptomatically beneficial drugs (21).

Data extraction. The following information was extracted from the studies: i) Name of first author and year of publication, and the method of generating the animal model; ii) sample size, sex, species and body weight of the animals; iii) timing and dosage of treatment as well as the treatment procedure; iv) outcome measures. If the outcome was evaluated at several time-points, the time-point of the last sacrifice was also extracted. The authors were requested to provide additional information if the data required for the review were incomplete or only presented graphically. When no response was received, digital ruler software was used to measure the data from the graphs. Data on the mean value and standard deviation were extracted for each treatment and control group. The time-point of lesion and drug administration were both set at zero.

Definitions of subgroups. It was expected that the numbers of TH-positive cells in the nigra would vary based on different animal strains and PD models. In the present review, the animals were classified into two groups. In one group, C57BL mice were injected with MPTP, while in the second group, Wistar rats were injected with 6-hydroxydopamine (6-OHDA).

Quality assessment. Methodological quality was assessed based on an eight-item modified scale from the STAIR list (21). The modified scale included the following items: i) Peer-reviewed publication; ii) sample size calculation; iii) randomization; iv) allocation concealment; v) report of animals excluded from analysis; vi) blinded assessment of the outcome; vii) compliance with animal welfare regulations; viii) report on potential conflicts of interest and funding sources. For calculation of the quality assessment aggregate score, each item on the eight-item scale was equal to one point.

Statistical analysis. Statistical analysis was performed using RevMan v.5.3 software (https://training.cochrane. org/online-learning/core-software-cochrane-reviews/revman). Publication bias was analyzed using STATA/SE 12.0 software (StataCorp). $\mathrm{P}<0.05$ was considered to indicate statistical significance. Data on TH-positive cells, pole test time and DA levels were considered continuous data. These indicators were used to estimate the combined effect size using the standardized mean difference (SMD). The SMD is utilized as a summary statistic in a meta-analysis when all studies assess the same outcome but measure it in different ways (22). Publication bias was assessed using a funnel plot and Egger's test (23). The $I^{2}$ statistic was used to assess heterogeneity. The fixed-effects model (Mantel-Haenszel method) was used if heterogeneity was negligible and the random-effects model (DerSimonian and Laird method) was used if heterogeneity was significant. To examine the robustness of the results, a sensitivity 


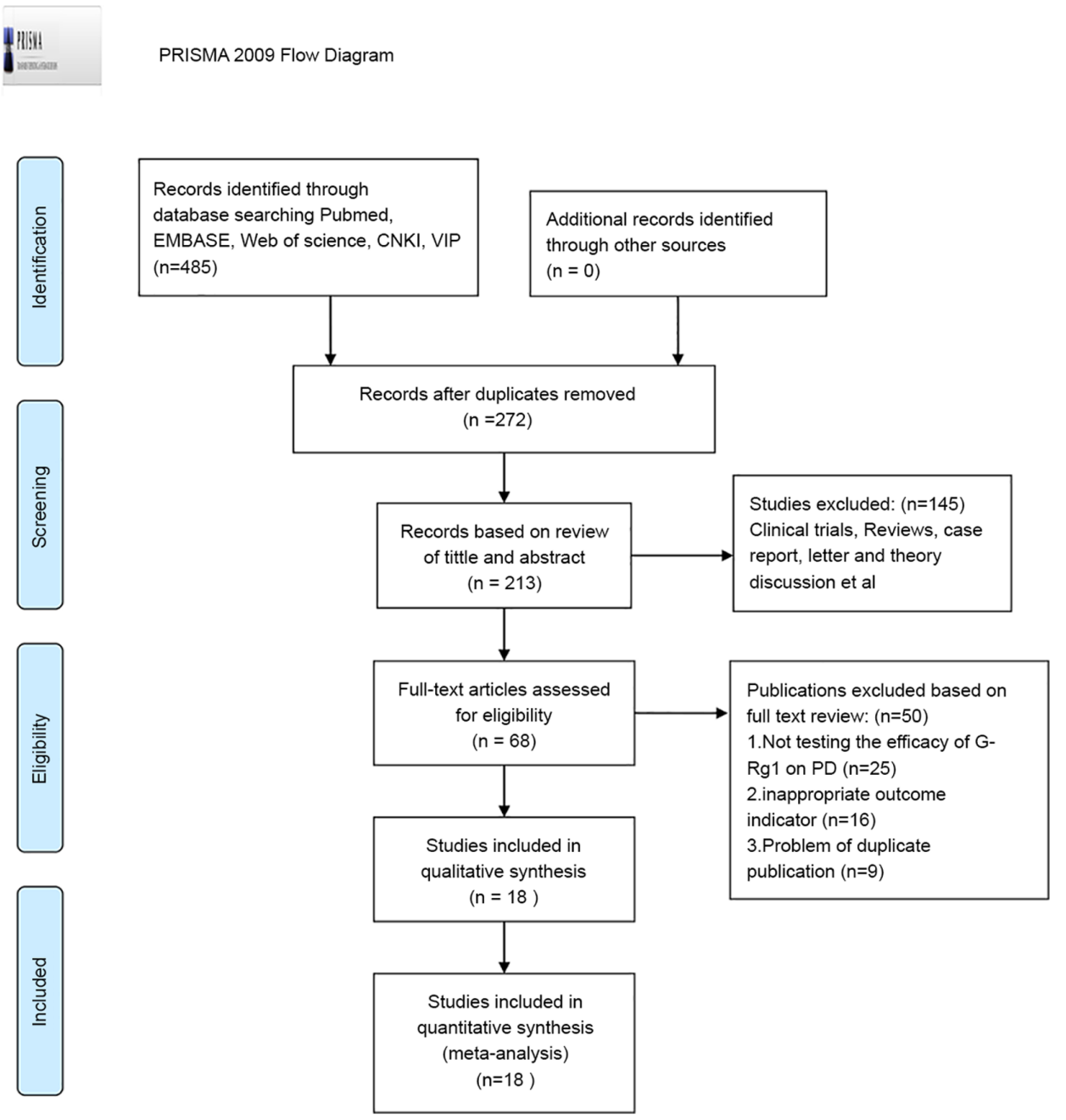

Figure 1. PRISMA flow diagram. PD, Parkinson's disease; G-Rg1, ginsenoside Rg1; PRISMA, Preferred Reporting Items for Systematic Reviews and Meta-Analyses; CNKI, Chinese National Knowledge Infrastructure.

analysis was performed by omitting each study in turn from the total and reanalyzing the quality of the remaining studies. Furthermore, the impact of factors influencing the outcome was evaluated using a pre-specified subgroup analysis based on the following features: Quality score, G-Rg1 dosage and animal weight. The difference between groups was measured by partitioning heterogeneity and using the $\chi^{2}$ distribution with n-1 degrees of freedom, where $n$ equals the number of groups. One-way ANOVA followed by Tukey's test was used to determine significance between groups by using GraphPad Prism 7.0 (GraphPad Software, Inc.).

\section{Results}

Characteristics of the included studies. following an independent review, 485 papers were identified. After removing duplicates, 213 unique articles were identified and 145 papers were excluded after reviewing the titles and abstracts due to at least one of the following reasons: i) Clinical trial and/or ii) review, case report, letter or theory discussion. After reading the remaining 68 papers, which reported the effect of G-Rg1 on PD models, 18 articles $(13,14,24-39)$ were identified as meeting the eligibility criteria (Fig. 1).
The studies involved a total of 343 animals (G-Rg1 group, $n=167$; control group, $n=176$ ) and they all belonged to two species: Wistar rats $(\mathrm{n}=18)(34,35)$ and C57BL/6 mice $(n=325)$. Furthermore, 16 out of the 18 studies used 1-methyl-4-phenyl-1,2,3,6-tetrahydropyridine (MPTP), -induced models, whereas the remaining two studies used 6-hydroxydopamine (6-OHDA)-induced models $(34,35)$. The sex of the animals used was male in 14 studies and female in 3 studies (34-36). One study did not report animal sex (25). All-female animals were ovariectomized and chloral hydrate and Euthanal were used in 4 studies and 1 study, respectively. The remaining 13 studies did not report the anesthetic drug used. The publication year of the studies ranged from 2002 to 2019 . The sample size used in the studies varied from 10 to 47 animals. The mice used weighed 16-30 g, while the rats weighed 220-250 g. However, only the mean or range of the data in each study was used for this meta-analysis, rather than individual data. The schedule of the MPTP injection differed, as $30 \mathrm{mg} / \mathrm{kg} / \mathrm{day}$ (d) intraperitoneally (i.p.) for $5 \mathrm{~d}$ was used in 13 studies $(13,24,33,37,38)$, $25 \mathrm{mg} / \mathrm{kg} / \mathrm{d}$ i.p. every $4 \mathrm{~d}$ on a $40-\mathrm{d}$ schedule was used in 1 study (14) and $60 \mathrm{mg} / \mathrm{kg} / \mathrm{d}$ i.p. for $1 \mathrm{~d}$ was used in 2 studies $(36,39)$. Treatment regimens included 2.5 and $3 \mu 1$ 


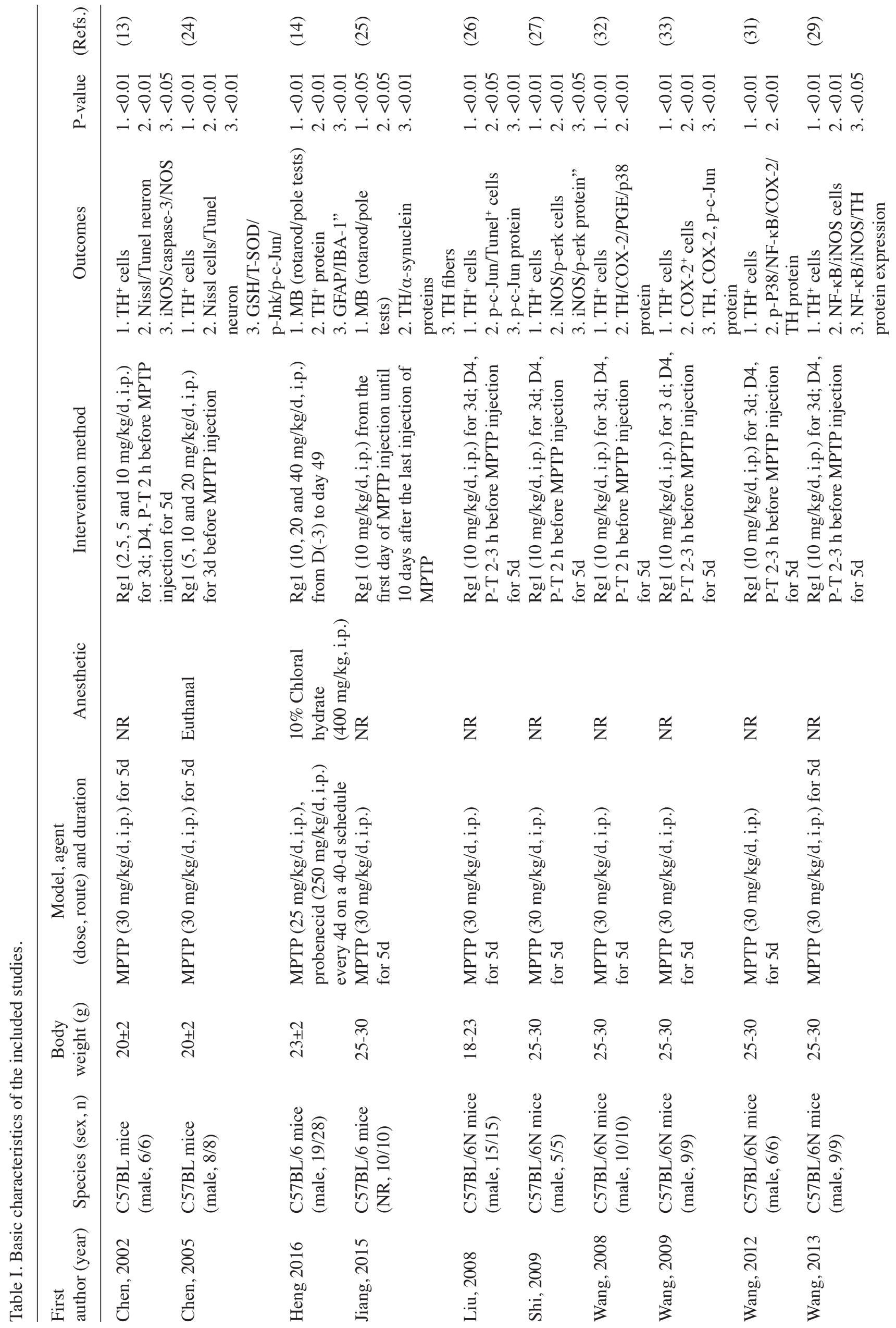




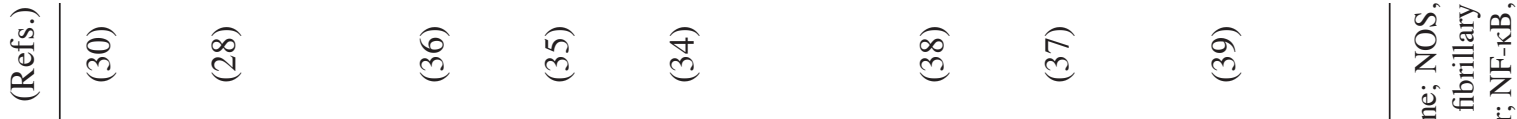

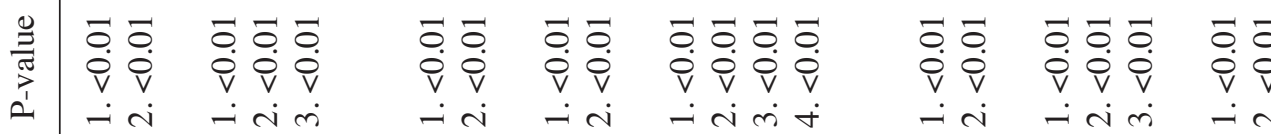

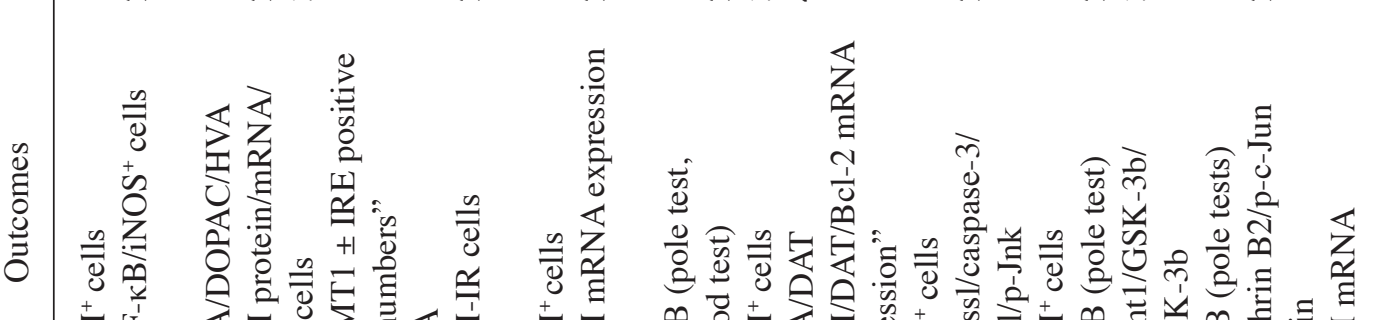

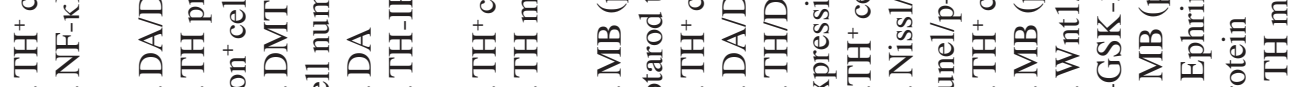
$-i$ i

मे $\dot{0} \stackrel{\overrightarrow{0}}{\overrightarrow{0}}$

焉

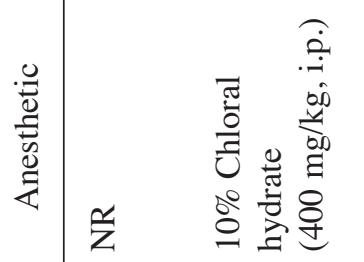

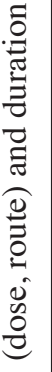

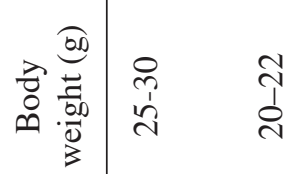

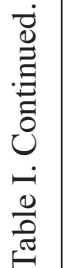

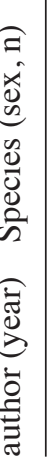

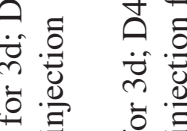

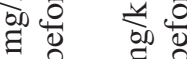

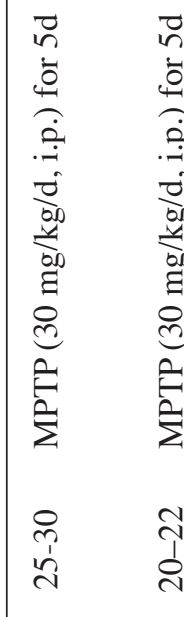

ڤิ)

$\sum$ 文

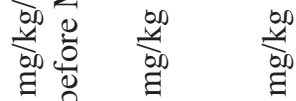

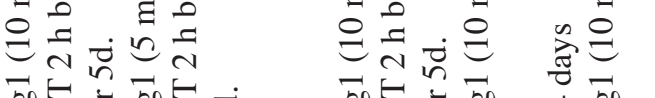

क人

ঙ.

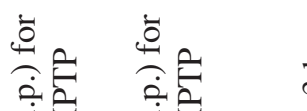

$\rightarrow \sum_{0} \rightarrow \sum_{0}$

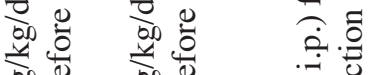

bD

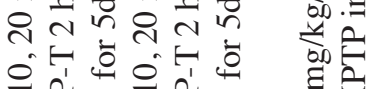

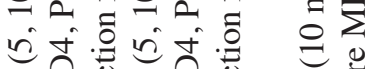

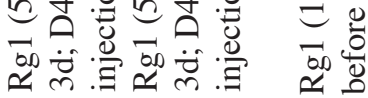

赔

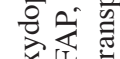

용

0
0
0

的

恶新

焉

:

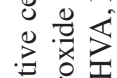

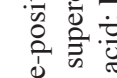

要

家客

政

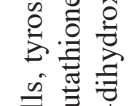

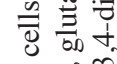

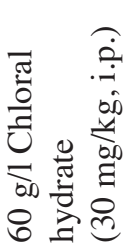

声武焉 元

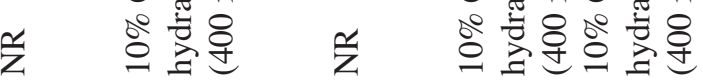

号学

В

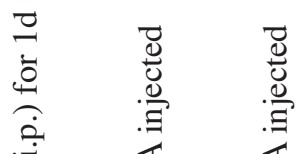

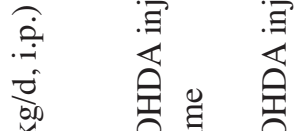

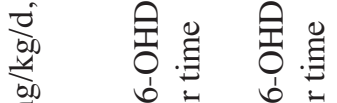

然

: m m m

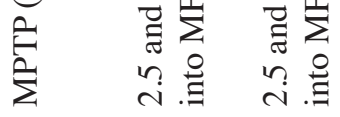

in $\quad$ in $\quad z$

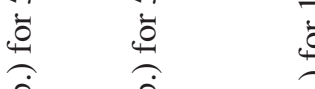

:

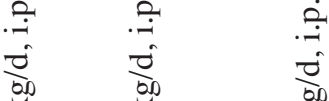

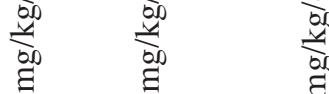

व ह व

言育

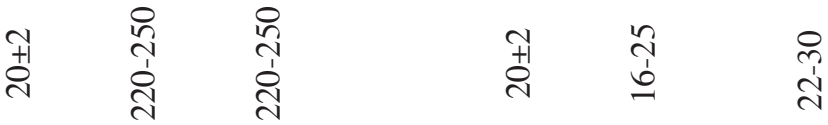

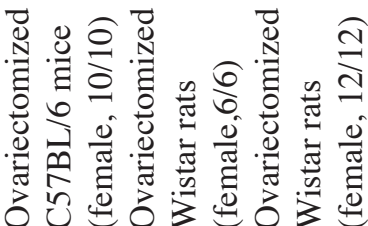

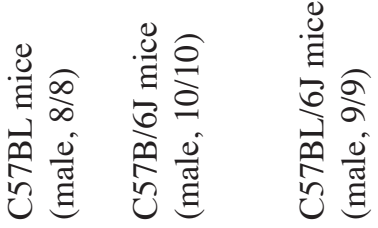

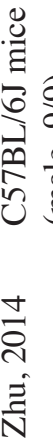

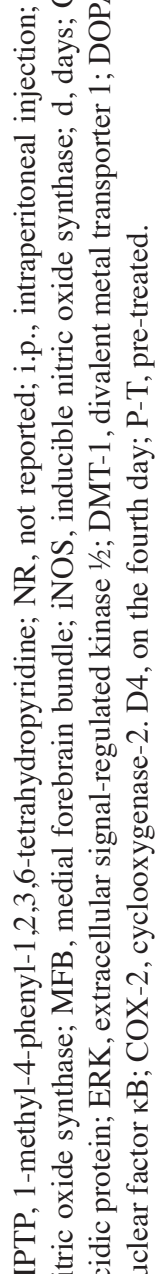


Table II. Quality assessment of included studies.

\begin{tabular}{|c|c|c|c|c|c|c|c|c|c|c|}
\hline \multirow[b]{2}{*}{ Author (year) } & \multicolumn{8}{|c|}{ Criterion no. } & \multirow[b]{2}{*}{ Total criteria fulfilled (n) } & \multirow[b]{2}{*}{ (Refs.) } \\
\hline & $\mathrm{i}$ & ii & iii & iv & $\mathrm{v}$ & vi & vii & viii & & \\
\hline Chen, 2002 & $\sqrt{ }$ & & $\sqrt{ }$ & & & $\sqrt{ }$ & $\sqrt{ }$ & & 4 & (13) \\
\hline Chen, 2005 & $\sqrt{ }$ & & $\sqrt{ }$ & $\sqrt{ }$ & & $\sqrt{ }$ & $\sqrt{ }$ & & 5 & (24) \\
\hline Heng, 2016 & $\sqrt{ }$ & $\sqrt{ }$ & $\sqrt{ }$ & $\sqrt{ }$ & $\sqrt{ }$ & $\sqrt{ }$ & $\sqrt{ }$ & $\sqrt{ }$ & 8 & (14) \\
\hline Jiang, 2015 & $\sqrt{ }$ & & $\sqrt{ }$ & $\sqrt{ }$ & & $\sqrt{ }$ & $\sqrt{ }$ & $\sqrt{ }$ & 6 & $(25)$ \\
\hline Liu, 2008 & $\sqrt{ }$ & & $\sqrt{ }$ & $\sqrt{ }$ & & $\sqrt{ }$ & $\sqrt{ }$ & & 5 & (26) \\
\hline Shi, 2009 & $\sqrt{ }$ & & $\sqrt{ }$ & & & & $\sqrt{ }$ & & 3 & $(27)$ \\
\hline Wang, 2008 & $\sqrt{ }$ & & $\sqrt{ }$ & & & $\sqrt{ }$ & $\sqrt{ }$ & & 4 & (32) \\
\hline Wang, 2009 & $\sqrt{ }$ & & $\sqrt{ }$ & & & $\sqrt{ }$ & $\sqrt{ }$ & & 4 & (33) \\
\hline Wang, 2012 & $\sqrt{ }$ & & $\sqrt{ }$ & & & & $\sqrt{ }$ & & 3 & $(31)$ \\
\hline Wang, 2013 & $\sqrt{ }$ & & $\sqrt{ }$ & $\sqrt{ }$ & & $\sqrt{ }$ & $\sqrt{ }$ & & 5 & $(29)$ \\
\hline Wang, 2014 & $\sqrt{ }$ & & $\sqrt{ }$ & $\sqrt{ }$ & $\sqrt{ }$ & & $\sqrt{ }$ & & 5 & $(30)$ \\
\hline Wang, 2009 & $\sqrt{ }$ & & $\sqrt{ }$ & $\sqrt{ }$ & & $\sqrt{ }$ & $\sqrt{ }$ & $\sqrt{ }$ & 6 & (28) \\
\hline Yan, 2014 & $\sqrt{ }$ & & $\sqrt{ }$ & & & $\sqrt{ }$ & $\sqrt{ }$ & & 4 & (36) \\
\hline $\mathrm{Xu}, 2008$ & $\sqrt{ }$ & & $\sqrt{ }$ & $\sqrt{ }$ & & $\sqrt{ }$ & $\sqrt{ }$ & $\sqrt{ }$ & 6 & (35) \\
\hline $\mathrm{Xu}, 2009$ & $\sqrt{ }$ & & $\sqrt{ }$ & & & $\sqrt{ }$ & $\sqrt{ }$ & & 4 & (34) \\
\hline Zhou, 2003 & $\sqrt{ }$ & & $\sqrt{ }$ & & & & $\sqrt{ }$ & & 3 & (38) \\
\hline Zhou, 2016 & $\sqrt{ }$ & & $\sqrt{ }$ & $\sqrt{ }$ & & $\sqrt{ }$ & $\sqrt{ }$ & $\sqrt{ }$ & 6 & (37) \\
\hline Zhu, 2014 & $\sqrt{ }$ & & $\sqrt{ }$ & & & $\sqrt{ }$ & $\sqrt{ }$ & & 4 & (39) \\
\hline
\end{tabular}

Criteria: i) A peer-reviewed publication; ii) sample size calculation; iii) randomization; iv) allocation concealment; v) reporting of animals excluded from analysis; vi) blinded assessment of outcome; vii) compliance with animal welfare regulations; viii) reporting potential conflicts of interest and study funding.

6-OHDA $(3.6 \mathrm{mg} / \mu \mathrm{l}$ in $0.9 \%$ saline containing $0.02 \% \mathrm{w} / \mathrm{v}$ ascorbic acid) unilaterally injected into the medial forebrain bundle per treatment in two studies $(34,35)$. A total of five studies used a dose gradient of G-Rg1. Among them, four studies used 5-, 10- and $20 \mathrm{mg} / \mathrm{kg}$ doses (24,36-38) and one study utilized 10-, 20- and 40-mg/kg doses (14), while the remaining studies applied 2.5-, 5- and 10-mg/kg doses (13). A total of 13 studies included a single dose of G-Rg1, $10 \mathrm{mg} / \mathrm{kg}$ in 12 studies $(25-27,29,36,39)$ and $5 \mathrm{mg} / \mathrm{kg}$ in 1 study (28). The number of TH-positive cells in the nigra was the outcome measure in 13 studies (13,24,26,27,29-35,37,38), Nissl-positive cells were the outcome measure in 3 studies $(13,23,37)$, the pole test time was the outcome measure in 4 studies $(14,25,37,39)$ and DA content in the striatum was the outcome measure in 3 studies $(28,34,36)$. In the 13 studies assessing the TH-positive nigra cells, the TH-positive cell count was appraised using diaminobenzidine staining before incubation with a TH polyclonal antibody and biotinylated $\operatorname{IgG}$ as the secondary antibody. The plasma and positive cell processes were stained brown and measured using analytical software. In the 3 studies with Nissl-positive cells as the outcome measure, the staining method was as follows: Paraffin sections were deparaffinized and hydrated, stained with methylene blue buffer for $10 \mathrm{~min}$ and immersed into an acetic acid buffer for $2 \mathrm{~min}$. The four studies in which the outcome was the pole test time employed a previously reported protocol (40). The total time required to climb down the pole was measured. The 3 studies in which the DA content in the striatum was the outcome measure, DA contents were measured by high-performance liquid chromatography with electrochemical detection and the results were expressed as $\mathrm{ng} / \mathrm{mg}$ wet weight of brain tissue (Table I).

Methodological quality. The quality scores of the 18 studies ranged from 3 to 8 , out of which three studies had a score of 3 , six studies had a score of 4 , four studies had a score of 5, four studies had a score of 6 and one study had a score of 8 (Table II). Only one study mentioned the sample size calculation. All studies presented detailed methods for the random allocation to the treatment group. Allocation concealment was performed in 9 studies. In addition, two studies reported conditions under which the animals were excluded from analysis, while 13 studies included a description of the blinded assessment of the outcome. Furthermore, five studies provided a statement of potential conflict of interest and funding sources (Table II).

Effectiveness. The analysis of TH-positive cells in the nigra included 204 animals in 13 studies, out of which 180 animals in 11 studies were included in the subgroup analysis of MPTP-induced mice and the remaining 24 animals in two studies were included in the subgroup analysis of 6-OHDA-induced rats. However, one study (25) was excluded from the pooled analysis because the data were presented in the form of percentages (TH-positive cells/control \%), and the means and standard deviations generated from the raw data were inaccessible. The whole data for analysis were 


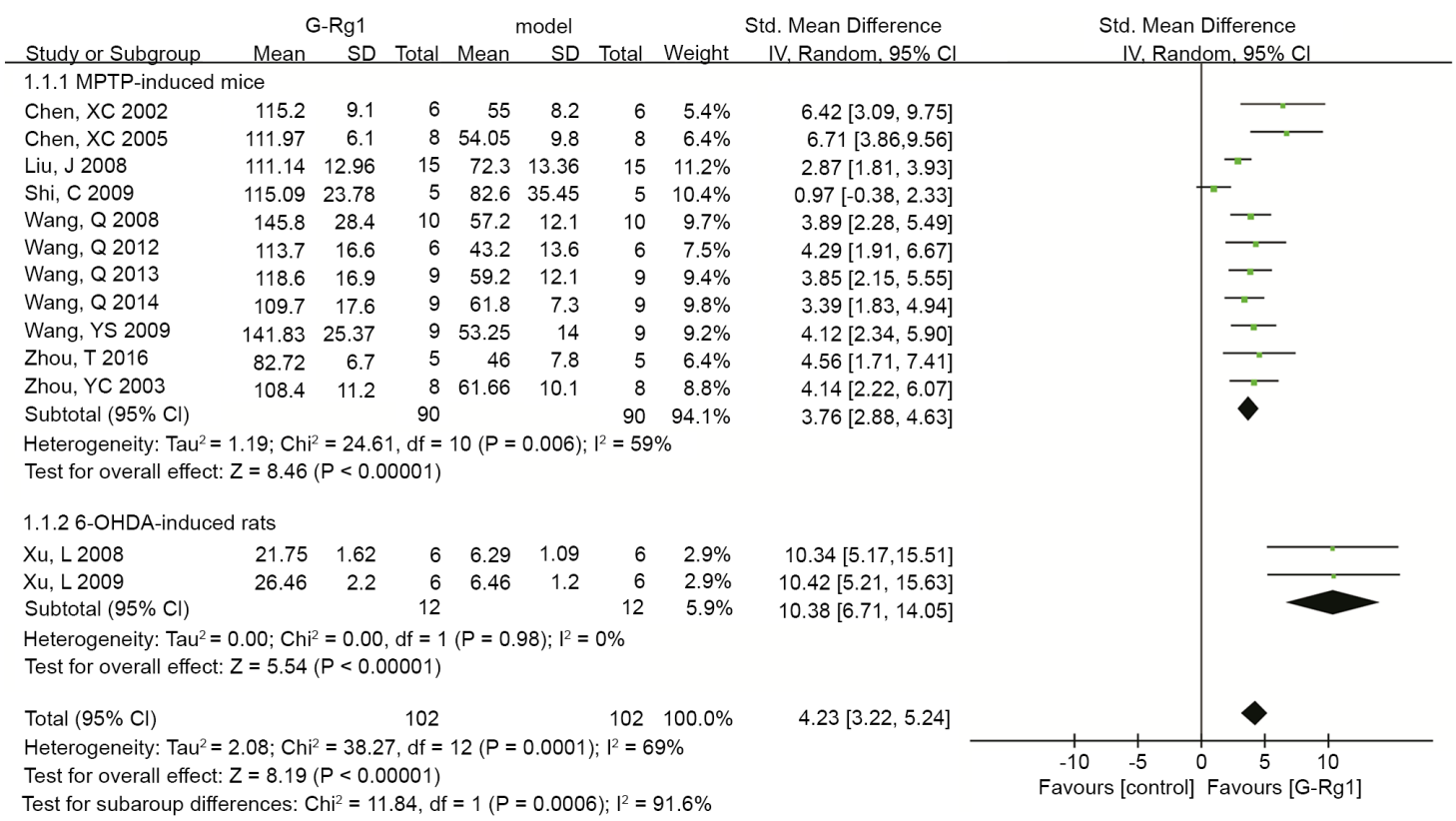

Figure 2. Pooled estimate of improvement in tyrosine hydroxylase-positive cells in the nigra. CI, confidence interval; df, degrees of freedom; SD, Std. deviation; Std., standard; IV, inverse variance; G-Rg1, ginsenoside Rg1; MPTP, 1-methyl-4-phenyl-1,2,3,6-tetrahydropyridine; OHDA, 6-hydroxydopamine.

pooled and a significant difference in the G-Rg1 treatment group compared to the control group was determined $(\mathrm{n}=204$, SMD: 4.23 , 95\% CI: 3.22 to 5.24, $\mathrm{P}<0.00001$; Fig. 2). The 6-OHDA-induced mice exhibited a larger effect size than the MPTP-induced mice (n=24, SMD: $10.38,95 \%$ CI: 6.71 to 14.05 vs. $n=180$, SMD: $3.76,95 \%$ CI: 2.88 to 4.63 , P<0.00001; Fig. 2). Furthermore, there was obvious heterogeneity between studies for the analysis of TH-positive cells in the MPTP-induced group $\left(\mathrm{Tau}^{2}=1.19, \mathrm{Chi}^{2}=24.61, \mathrm{P}=0.006, \mathrm{I}^{2}=59 \%\right.$; Fig. 2$)$. The results on $\mathrm{TH}$-positive cells and heterogeneity were inconsistent after sequentially excluding each of the studies. The outlier studies (27) were excluded to produce more homogeneous results $\left(\mathrm{Tau}^{2}=0.13, \mathrm{Chi}^{2}=10.29, \mathrm{P}=0.33, \mathrm{I}^{2}=13 \%\right)$ and $\mathrm{a}$ larger effect size ( $n=170$, SMD: $3.89,95 \%$ CI: 3.26 to 4.51 , $\mathrm{P}<0.00001)$ in the subgroup analysis of MPTP-induced mice. In addition, 3 studies revealed significant effects of G-Rg1 on Nissl-positive cells compared with the control group $(n=44$, SMD: 15.03 , 95\% CI: 11.28 to $18.78, \mathrm{P}<0.00001$; heterogeneity: $\mathrm{Chi}^{2}=0.99, \mathrm{P}=0.61, \mathrm{I}^{2}=0 \%$; Fig. 3). Furthermore, four studies reported that $\mathrm{G}-\mathrm{Rg} 1$ decreased the pole test time compared to the control group $(n=105$, SMD: $-2.08,95 \%$ CI: -3.91 to -0.24 , $\mathrm{P}=0.03$; heterogeneity: $\mathrm{Tau}^{2}=3.02, \mathrm{Chi}^{2}=34.81, \mathrm{P}<0.00001$, $\mathrm{I}^{2}=91 \%$; Fig. 4), and the data were stable based on sensitivity analysis. In three studies, the DA levels in the striatum were indicated to be significantly improved in the G-Rg1 group compared with those in the control group (n=44, SMD: 2.71 , 95\% CI: 1.80 to $3.63, \mathrm{P}<0.00001$; heterogeneity: $\mathrm{Chi}^{2}=2.60$, $\mathrm{P}=0.27, \mathrm{I}^{2}=23 \%$; Fig. 5). The funnel plot indicated mild publication bias concerning the outcome of TH-positive cells upon visual inspection (Fig. 6A). In addition, Egger's test revealed moderate publication bias for the studies with $\mathrm{TH}$-positive cells as the outcome ( $\mathrm{P}=0.014$; Fig. $6 \mathrm{~B})$.

Pre-specified subgroup analysis. In the subgroup analysis for the outcome measure of TH-positive cells, the effect size of G-Rg1 in low-quality studies (quality score=3) was much smaller than that in higher-quality studies (Fig. 7A) $(\mathrm{P}<0.05)$. The G-Rg1 dose effect on TH-positive cells was then investigated. A high dose of G-Rg1 (20 mg/kg; n=26, 3 studies) was more effective at increasing dopaminergic neurons than a moderate dose $(10 \mathrm{mg} / \mathrm{kg} ; \mathrm{n}=180,11$ studies $)(\mathrm{P}<0.05)$, low dose $(5 \mathrm{mg} / \mathrm{kg} ; \mathrm{n}=32,4$ studies $)$ or very low dose $(2.5 \mathrm{mg} / \mathrm{kg}$; n=6, 1 study; Fig. 7B $(\mathrm{P}<0.05)$. Of note, the effect size was observed to be higher in younger mice (body weight, 16-25 g) than in older mice (body weight, 25-30 g) $(\mathrm{P}<0.05)$. Based on effect size, rats (body weight, 220-250 g) were preferable to mice (body weight, 16-30 g; Fig. 7C) $(\mathrm{P}<0.05)$.

\section{Discussion}

The present study provided a systematic review and meta-analysis to explore the effect of G-Rg1 in animal models of PD. A total of 18 studies with the outcomes of TH-positive cells in the nigra, total pole test time and DA contents in the striatum indicated significant improvement in animal models of PD after G-Rg1 treatment. The present meta-analysis revealed that pretreatment with specific doses of G-Rg1 is able to minimize the loss of dopaminergic neurons in both the nigra and the striatum and improve motor function in animal models of PD.

Methodological quality was assessed based on an eight-item modified scale from the STAIR list. The quality scores of the 18 studies ranged from 3 to 8 . High-quality papers (quality scores $\geq 6)(14,25,28,35,37)$ are more rigorous in their research design and they generally included sample size calculation, allocation concealment, reporting of animals excluded from analysis, potential conflicts of interest and funding in their study. A total of three studies $(27,31,38)$ had poor quality (quality scores=3) and they were peer-reviewed publications featuring randomization and compliance with animal welfare regulations, but did not describe the sample size calculation, allocation concealment, reporting of animals excluded from analysis, blinded assessment of outcomes, reporting potential 


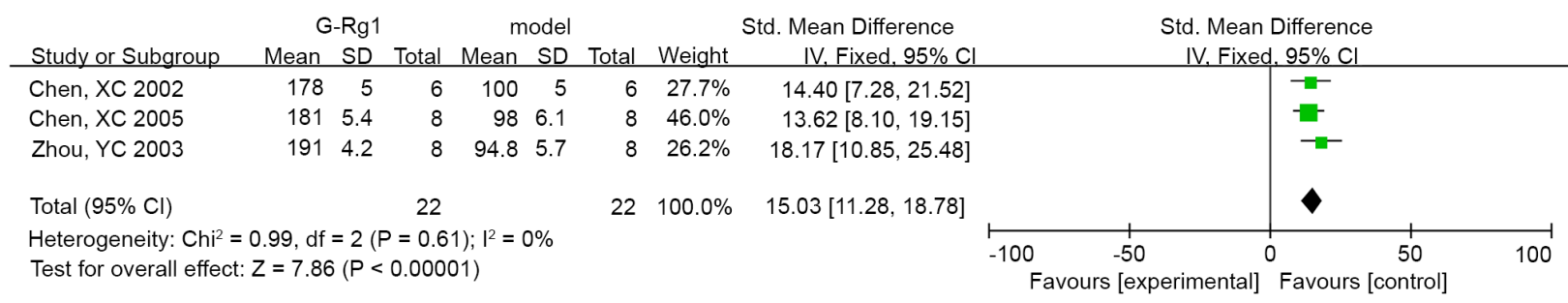

Figure 3. Pooled estimate of improvement in Nissl-positive cells in the nigra. CI, confidence interval; df, degrees of freedom; SD, Std. deviation; Std., standard; IV, inverse variance; G-Rg1, ginsenoside Rg1.

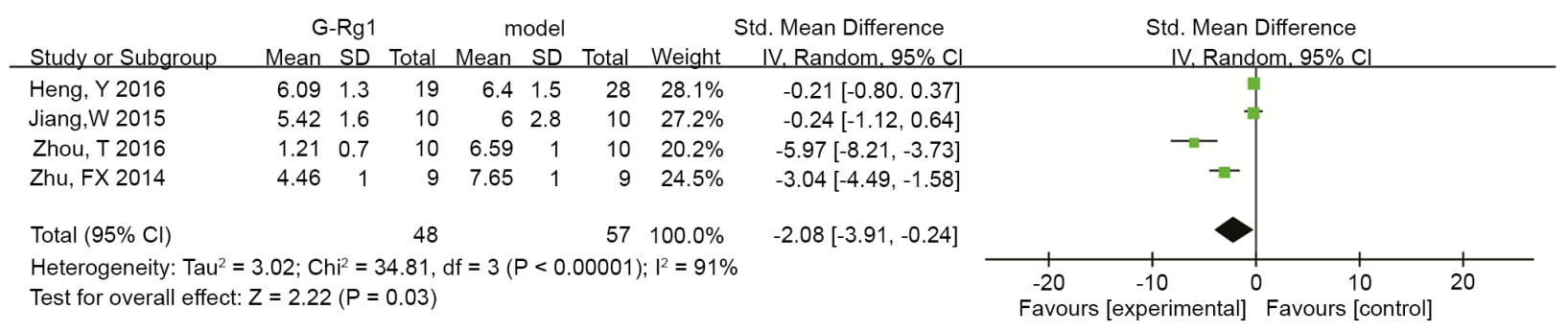

Figure 4. Pooled estimate of decrement in total time of pole test. CI, confidence interval; df, degrees of freedom; SD, Std. deviation; Std., standard; IV, inverse variance; G-Rg1, ginsenoside $\operatorname{Rg} 1$.

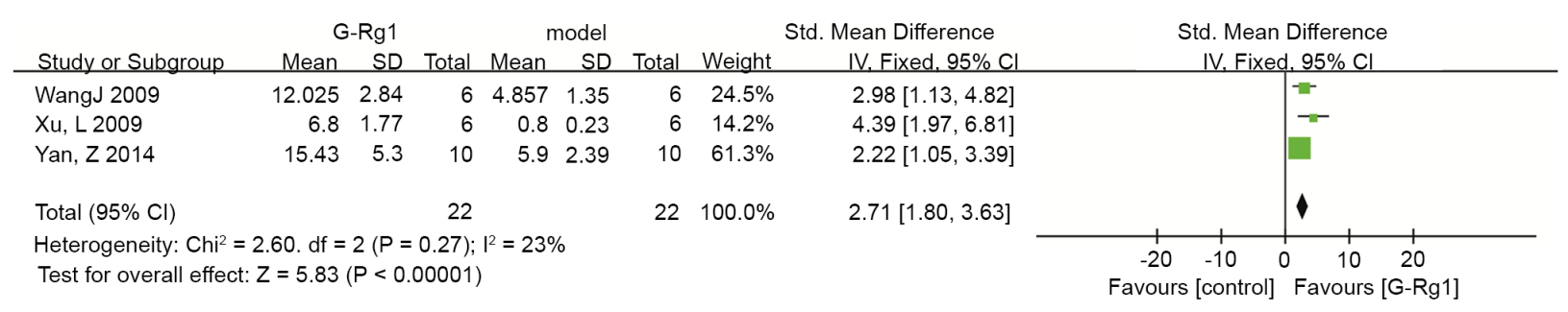

Figure 5. Pooled estimate of improvement in dopamine levels in the striatum. CI, confidence interval; df, degrees of freedom; SD, Std. deviation; Std., standard; IV, inverse variance; G-Rg1, ginsenoside Rg1.

conflicts of interest and study funding, which may decrease the reliability of the results.

Several limitations of the present study should be considered. First, seven papers were included in English-language databases (PubMed, EMBASE and Web of Science) and the remaining 11 papers were published in the Chinese language, which may lead to selection bias. Furthermore, the present analysis focused on animal studies, as no published studies were reporting on clinical trials of G-Rg1 treatment for PD and data from clinical studies are more valuable than those from animal studies. In addition, the included studies in the present meta-analysis did not report any negative results on TH-positive cells in the nigra, DA levels in the striatum or pole test time. There may have been an overestimation of the results because only available data were included in the analysis, and articles with negative results are more difficult to publish. In addition, the treatment regimens of $\operatorname{Rg} 1$ in the included studies varied widely (e.g. in terms of pre- and post-treatment to MTPT or 6-OHDA, frequency and duration), which was also a limitation of the present study. As another limitation, only the association, rather than causation, was evaluated, since the present meta-analysis was an observational research study rather than being experimental. None of the studies reported the effect of G-Rg1 in PD in other species, such as primates. Furthermore, there is no standard way to produce and most importantly assess dopaminergic lesions following toxin-induced lesion in rodents and the complexity includes the dose, the method used to assess denervation and the timing of the assessment after intoxication. In the present study, the SMD rather than the normalized mean difference (NMD) was used. However, SMD is more conservative than the NMD (22). Finally, among the studies included in the present meta-analysis, mild publication bias was suggested by the funnel plot and Egger's test. Studies with non-significant results may remain unpublished because the authors do not submit their manuscripts to journals for publishing, resulting in potential publication bias. Selective publishing and reporting also contribute to this bias, which must be considered. However, in the present study, publication bias was a possible explanation because all of the 18 included studies had positive rather than negative results.

Significant differences were observed between high- and low-quality papers based on the outcome measures. Specifically, for TH-positive cell outcomes, low-quality studies indicated the lowest effect of G-Rg1, suggesting that a poor-quality research design may have influenced the outcomes of certain previous 


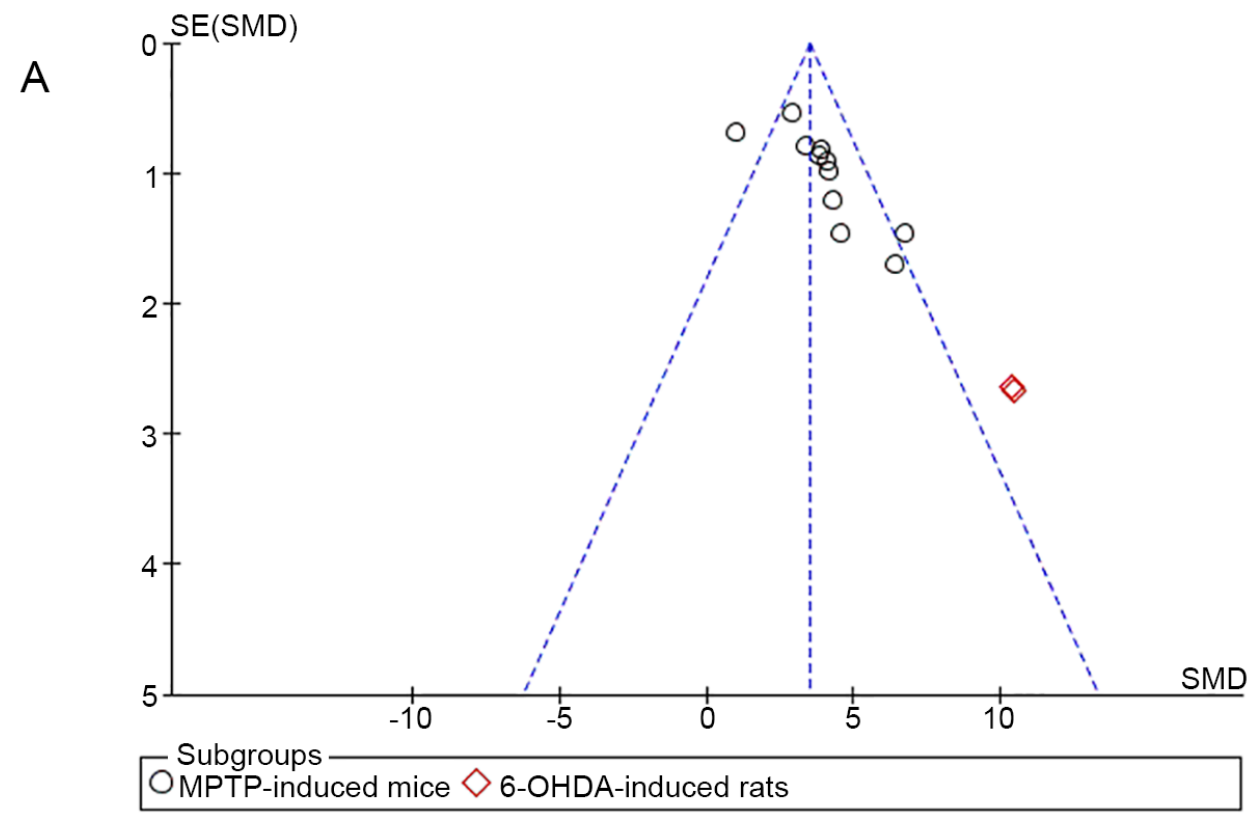

B

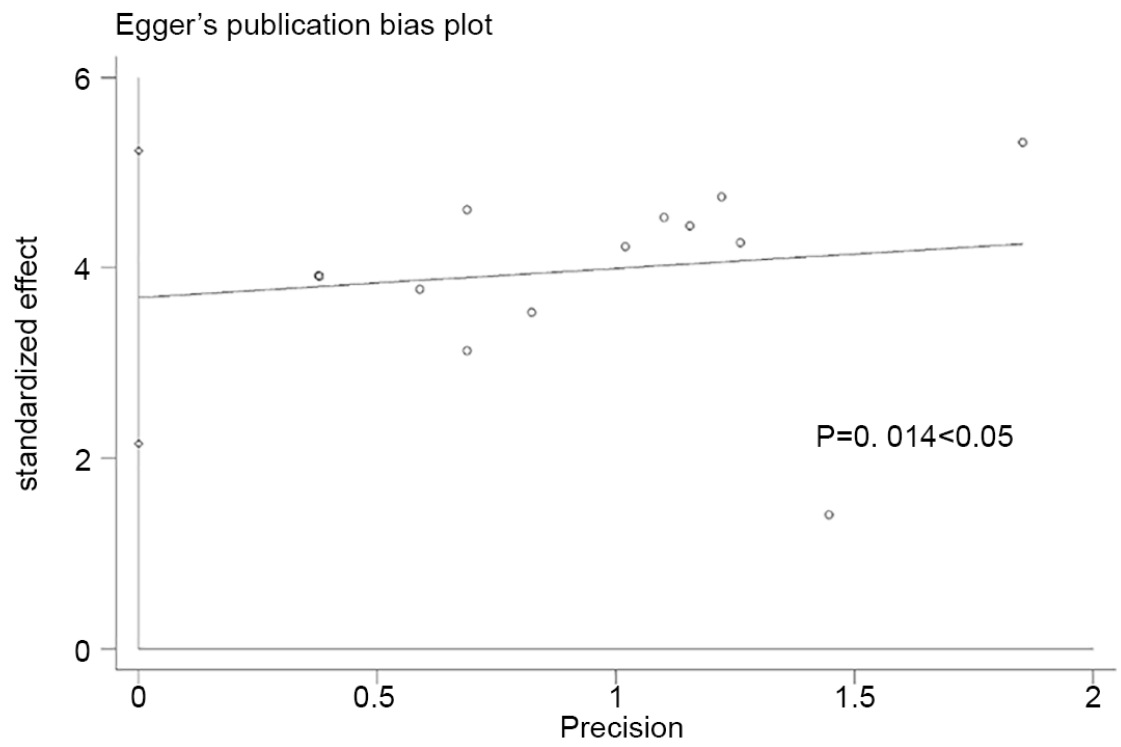

Figure 6. Bias assessment plot for the effect of ginsenoside Rg1 on tyrosine hydroxylase-positive cells by (A) funnel plot and (B) Egger's test. SE, standard error; SMD, standardized mean difference; MPTP, 1-methyl-4-phenyl-1,2,3,6-tetrahydropyridine; OHDA, 6-hydroxydopamine.

studies (41). High-quality studies may have a lower variance, which increases the effect size. On the other hand, improving the quality of studies may help reduce bias when such trials are included in meta-analyses. However, when the data from lower-quality trials are used in meta-analyses, the treatment efficacy may be statistically exaggerated by $30-50 \%$ (42). This may explain why the effect size of moderate-quality studies (quality score $=4$ ) is slightly higher than that of higher-quality studies (quality score $=5$ ). Certain scholars consider allocation concealment or randomization to be the major factors that inflate estimates of treatment efficacy (43). Consequently, high-quality, well-designed studies are required to determine the efficacy of G-Rg1 in PD. Based on the effect size, a high dose of G-Rg1 (20 mg/kg) was indicated to have the highest efficacy in PD models. However, only three studies used this dosage and 13 studies used a dose of $10 \mathrm{mg} / \mathrm{kg}$ to examine the outcome of TH-positive cells in the nigra. Therefore, these results should be interpreted with caution in this subgroup analysis. The effects of different dosages of G-Rg1, including their toxic effects, should be explored in future studies. In the present meta-analysis, according to the effect size, the efficacy of G-Rg1 to improve dopaminergic neurons was better in the 6-OHDA-induced rats than in the MPTP-induced mice. However, these results should be interpreted with caution as, in the present meta-analysis, 16 studies used the MPTP-induced model, whereas only 2 studies used 6-OHDA treatment of rats to induce the model of PD. By now, there is sufficient literature illustrating the neuroprotective effects of G-Rg1 in animal models (15). Therefore, in future research, more evidence should be gathered regarding the efficacy of G-Rg1 in 6-OHDA-induced rats. Only 3 studies included in the present meta-analysis measured Nissl stain-positive cells, which may directly indicate the survival of neurons. Loss of TH expression is not necessarily related to cells dying $(16,44)$, and following MPTP and 6-OHDA treatment, 
A

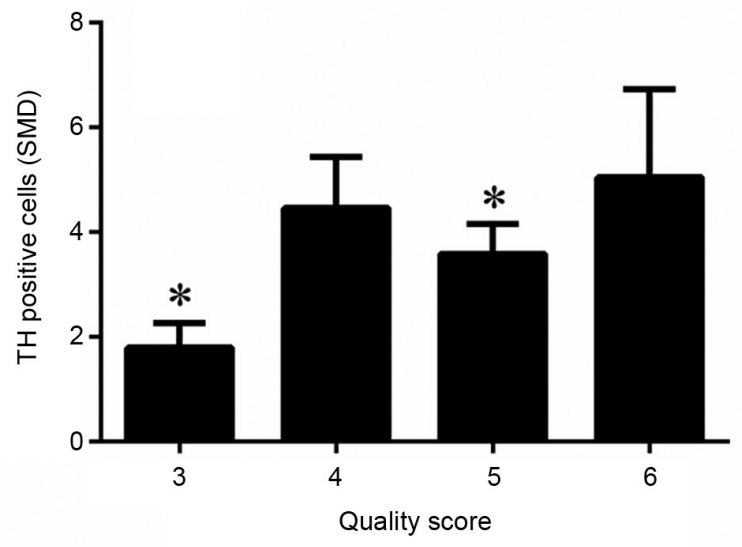

C

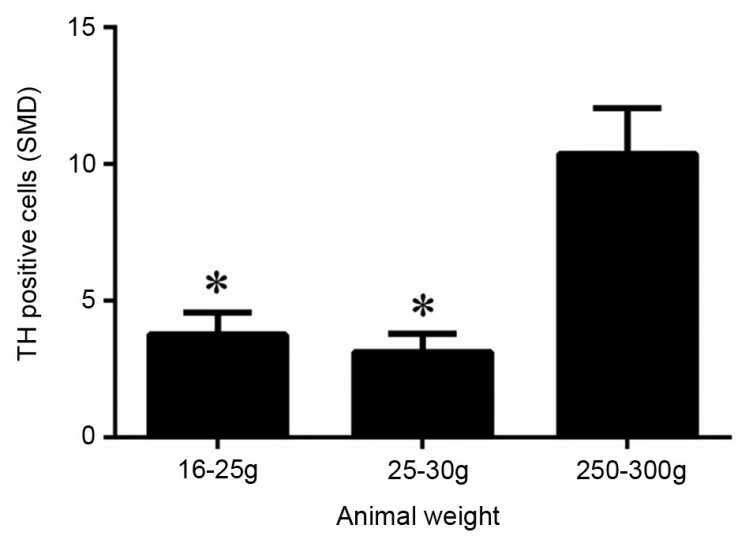

B

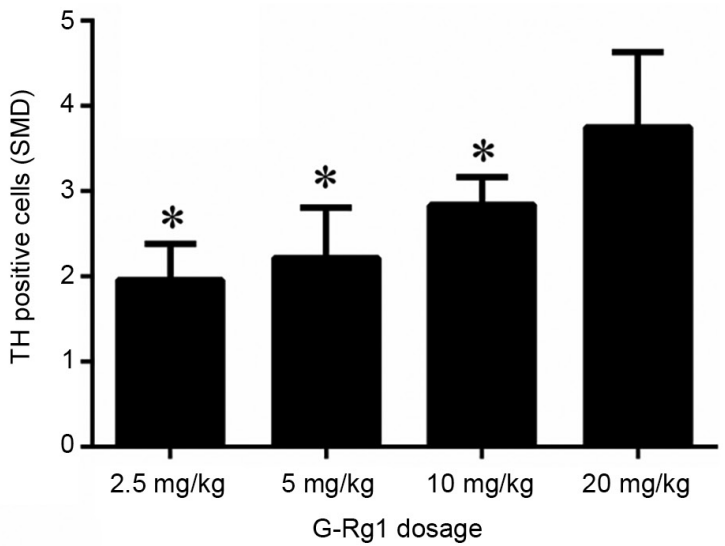

Figure 7. Subgroup analysis according to TH-positive cells. (A) Quality score. * $\mathrm{P}<0.05$ compared with quality score 6 group. (B) G-Rg1 dosage. * $<<0.05$ compared with the $20 \mathrm{mg} / \mathrm{kg}$ group. (C) Animal weight. ${ }^{*} \mathrm{P}<0.05$ compared with the $250-300 \mathrm{~g}$ group. The vertical error bars represent the effect size of G-Rg1 and the error bars represent standard deviations for each group in the subgroup analysis. TH, tyrosine hydroxylase; G-Rg1, ginsenoside Rg1; SMD, standardized mean difference.

there is a temporal association of tyrosine nitration or cysteine oxidation with inactivation of $\mathrm{TH}$ in vitro, suggesting that this covalent post-translational modification is responsible for the in vivo loss of $\mathrm{TH}$ neurons (45-47). Nissl staining may make the conclusions more stable in experimental models of PD and future research should pay close attention to this. Furthermore, no published papers are exploring the joint action of G-Rg1 with other neuroprotectants on PD, which should be investigated in future clinical trials. To the best of our knowledge, there are still no clinical studies reporting the effects of G-Rg1 on PD. However, the above results suggest a potential treatment effect of G-Rg1 on PD suitable for a clinical study.

The studies included in the present meta-analysis that used the MPTP-induced model did not strictly follow the protocol of Jackson-Lewis and Przedborski from 2007 (48). Besides, the studies reported on whether G-Rg1 interfered with MPTP toxicokinetics and pre-treatment or if co-administration with G-Rg1 invalidated the interpretation of the data. It is also uncertain whether G-Rg1 prevented the uptake of MPTP by blocking DAT (DA transporter), preventing the conversion of MPTP to MPP and detoxifying MPTP. Therefore, the method of pretreatment with G-Rg1 may not be scientific (48). Further studies on the pharmacological relationship between G-Rg1 and MPTP are required. Furthermore, all studies in the present meta-analysis counted the cell numbers immediately after the last injection of MPTP. This may have led to the reporting of higher numbers as cells take time to die and the best option is to determine the cell numbers after 15 days of toxin application $(16,44)$.

No obvious toxicity of G-Rg1 toward the animals was observed in the 18 studies analyzed in the present study. However, one study that included toxicity testing reported that the intravenous median lethal dose of the combination of salvianolic acid B (SalB) and G-Rg1 was $1,747 \mathrm{mg} / \mathrm{kg}$. This dose was 100 -fold greater than the effective dose (15 mg/mg), suggesting that SalB-Rg1 and G-Rg1 is a safe combination that may be considered for future clinical development (49). However, the safety of intravenous ginsenoside-Rg1 calls for extensive basic research and large-scale clinical trials.

Ongoing research is investigating the proposed mechanisms of G-Rg1, including the stimulation of antioxidants (50), anti-inflammatory (51), anti-apoptotic (52) and immune activities (53), the potentiation of nerve growth factor activity (54), maintenance of cellular ATP levels (55), inhibition of excitotoxicity (56), $\mathrm{Ca}^{2+}$ over-influx into neurons (57) and the preservation of the structural integrity of neurons (58). The possible mechanisms underlying the effects of G-Rg1 should be further investigated in future studies.

In conclusion, G-Rg1 was able to attenuate the damage caused by toxicants in the nigra and the striatum, as evidenced 
by increased numbers of TH-positive cells and DA levels in the striatum and of Nissl-positive cells, and improved motor function associated with a reduction in the total pole test time in animal models of PD. G-Rg1 has positive effects in attenuating damage in models of PD, suggesting that it is a possible candidate neuroprotective drug for treating human PD. However, there is potential publication bias in most of the reported studies and the limited quality of the experiments decreases the reliability of these results. Further studies should confirm if the neuroprotectant $\mathrm{G}-\mathrm{Rg} 1$ is a promising drug candidate for PD.

\section{Acknowledgements}

Not applicable.

\section{Funding}

The current study was supported by the National TCM Foundation of China (grant no. JDZX2015111), the Zhejiang Science Foundation of China (grant nos. LY14H080004 and LY15H040011) and the Projects of the Zhejiang Committee of Chinese medicine, China (grant no. 2013ZB045).

\section{Availability of data and materials}

All data generated or analyzed during this study are included in this published article.

\section{Authors' contributions}

YBH, YS and ZDY designed the current study, searched databases, extracted and assessed the literature and drafted the manuscript. YBH, JHL and YMG statistically analyzed the data. YBH and YMC confirm the authenticity of all the raw data. YMC and YLL conceived and designed the present study, provided general supervision and finalized the manuscript. All authors read and approved the final manuscript.

\section{Ethics approval and consent to participate}

Not applicable.

\section{Patient consent for publication}

Not applicable.

\section{Competing interests}

The authors declare that they have no competing interests.

\section{References}

1. Barnett R: Parkinson's disease. Lancet 387: 217, 2016.

2. Toulouse A and Sullivan AM: Progress in Parkinson's disease-where do we stand? Prog Neurobiol 85: 376-392, 2008

3. Schapira AH: Molecular and clinical pathways to neuroprotection of dopaminergic drugs in Parkinson disease. Neurology 72 (Suppl 7): S44-S50, 2009.

4. Beal MF: Bioenergetic approaches for neuroprotection in Parkinson's disease. Ann Neurol 53 (Suppl 3): S39-S48, 2003.

5. Olanow CW and Schapira AH: Therapeutic prospects for Parkinson disease. Ann Neurol 74: 337-347, 2013.
6. Schapira AH: Treatment options in the modern management of Parkinson disease. Arch Neurol 64: 1083-1088, 2007.

7. Ellis JM and Reddy P: Effects of Panax ginseng on quality of life. Ann Pharmacother 36: 375-379, 2002.

8. Rausch WD, Liu S, Gille G and Radad K: Neuroprotective effects of ginsenosides. Acta Neurobiol Exp (Wars) 66: 369-375, 2006.

9. Ong WY, Farooqui T, Koh HL, Farooqui AA and Ling EA: Protective effects of ginseng on neurological disorders. Front Aging Neurosci 7: 129, 2015.

10. Chen XC, Fang F, Zhu YG, Chen LM, Zhou YC and Chen Y: Protective effect of ginsenoside Rg1 on $\mathrm{MPP}^{+}$-induced apoptosis in SHSY5Y cells. J Neural Transm (Vienna) 110: 835-845, 2003.

11. Radad K, Gille G, Moldzio R, Saito H, Ishige K and Rausch WD: Ginsenosides Rb1 and Rg1 effects on survival and neurite growth of $\mathrm{MPP}^{+}$-affected mesencephalic dopaminergic cells. J Neural Transm (Vienna) 111: 37-45, 2004.

12. Radad K, Gille G, Moldzio R, Saito H and Rausch WD: Ginsenosides Rb1 and Rg1 effects on mesencephalic dopaminergic cells stressed with glutamate. Brain Res 1021: 41-53, 2004.

13. Chen XC, Chen Y, Zhu YG, Fang F and Chen LM: Protective effect of ginsenoside Rg1 against MPTP-induced apoptosis in mouse substantia nigra neurons. Acta Pharmacol Sin 23: 829-834, 2002.

14. Heng Y, Zhang QS, Mu Z, Hu JF, Yuan YH and Chen NH: Ginsenoside Rg1 attenuates motor impairment and neuroinflammation in the MPTP-probenecid-induced parkinsonism mouse model by targeting $\alpha$-synuclein abnormalities in the substantia nigra. Toxicol Lett 243: 7-21, 2016.

15. Song L, Xu MB, Zhou XL, Zhang DP, Zhang SL and Zheng GQ: A preclinical systematic review of ginsenoside-Rg1 in experimental Parkinson's disease. Oxid Med Cell Longev 2017: 2163053, 2017.

16. Stanic D, Finkelstein DI, Bourke DW, Drago J and Horne MK: Timecourse of striatal re-innervation following lesions of dopaminergic SNpc neurons of the rat. Eur J Neurosci 18: 1175-1188, 2003.

17. Moher D, Liberati A, Tetzlaff J and Altman DG; PRISMA Group: Preferred reporting items for systematic reviews and meta-analyses: The PRISMA statement. Int J Surg 8: 336-341, 2010.

18. Fifel $\mathrm{K}$ and Cooper HM: Loss of dopamine disrupts circadian rhythms in a mouse model of Parkinson's disease. Neurobiol Dis 71: 359-369, 2014.

19. Morin N, Jourdain VA and Di Paolo T: Modeling dyskinesia in animal models of Parkinson disease. Exp Neurol 256: 105-116, 2014.

20. Matsumoto M: Dopamine signals and physiological origin of cognitive dysfunction in Parkinson's disease. Mov Disord 30: 472-483, 2015

21. Jagmag SA, Tripathi N, Shukla SD, Maiti S and Khurana S: Evaluation of models of Parkinson's disease. Front Neurosci 9: 503,2016

22. Vesterinen HM, Sena ES, Egan KJ, Hirst TC, Churolov L, Currie GL, Antonic A, Howells DW and Macleod MR: Meta-analysis of data from animal studies: A practical guide. J Neurosci Methods 221: 92-102, 2014.

23. Egger M, Davey Smith G, Schneider M and Minder C: Bias in meta-analysis detected by a simple, graphical test. BMJ 315 : 629-634, 1997.

24. Chen XC, Zhou YC, Chen Y, Zhu YG, Fang F and Chen LM: Ginsenoside Rg1 reduces MPTP-induced substantia nigra neuron loss by suppressing oxidative stress. Acta Pharmacol Sin 26: 56-62, 2005.

25. Jiang W, Wang Z, Jiang Y, Lu M and Li X: Ginsenoside Rg1 ameliorates motor function in an animal model of Parkinson's disease. Pharmacology 96: 25-31, 2015.

26. Liu J, Li R, Liu LN, Zhang XW, Zhang YX and Zhang ZF: Protective effect of ginsenoside Rg1 on jnk signaling pathway mediatethe loss of nigral nurons in the mice model of Parkinson. Mod Prev Med 35: 1973-1975, 2008 (In Chinese).

27. Shi C,Zhang YX and Zhang ZF: Effect of phosphorylated-ERK1/2 on inducible nitric oxide synthase expression in the substantia nigra of mice with MPTP-induced Parkinson disease. Nan Fang Yi Ke Da Xue Xue Bao 29: 60-63, 2009 (In Chinese).

28. Wang J, Xu HM, Yang HD, Du XX, Jiang H and Xie JX: Rg1 reduces nigral iron levels of MPTP-treated C57BL6 mice by regulating certain iron transport proteins. Neurochem Int 54: 43-48, 2009.

29. Wang Q, Zhang $\mathrm{H}$ and Liu M: Influence of NF- $\kappa \mathrm{B}$ on i-NOS expression in substantia nigra of mouse models of Parkinson's disease induced by MPTP. J Hebei United Univ 15: 297-299, 2013 (In Chinese). 
30. Wang Q, Zhang H, Liu M, Li QJ, Geng LX, Sun MH, Tian QY and Zhang YX: Influence of ginsenoside Rg1 in expressions of FADD and FLIP in substantia nigra of Parkinson's disease model mice. J Jilin Univ Med Ed 40: 962-966, 2014.

31. Wang Q, Zhang H, Zhang ZF, Wei ZF, Wang YS, Zhou HX, et al: Role of P38 MAPK in regulating expression of NF- $\kappa \mathrm{B}$ and COX-2 in substantia-nigra of MPTP Parkinson's disease mice model. China J Mod Med 22: 15-20, 2012 (In Chinese).

32. Wang Q, Zhang YX and Zhang ZF: Influence of NF- $\kappa B$ on COX-2 expression insubstantia nigra of mouse models of Parkinson's disease induced by MPTP. J Fourth Mil Med Univ 29: 1757-1760, 2008.

33. Wang YS, Li H, Zhang YX, Wei SP, Zhang ZF and Tian QY: Influence of ginsenoside Rg1 on p-c-jun and cox-2 epression in substantia nigra of the MPTP mouse model of subacute Parkinson's disease. Chin J Neuroanat 25: 432-436, 2009.

34. Xu L, Chen WF and Wong MS: Ginsenoside Rg1 protects dopaminergic neurons in a rat model of Parkinson's disease through the IGF-I receptor signalling pathway. Br J Pharmacol 158 : 738-748, 2009.

35. Xu L, Liu LX, Chen LX, Xie JX and Huang WX: The protective effect of ginsenoside Rg1 on dopaminergic neurons of substantia in the ovariectomized rat model of Parkinson's disease. Zhongguo Ying Yong Sheng Li Xue Za Zhi 24: 1-5, 2008 (In Chinese).

36. Yan Z, Wu L, Xue D, Gao XQ and Chen WF: Effects of gesenoside $\mathrm{Rg} 1$ and insulin-like growth factor on dopaminergic neurons in Parkinson. Acta Acad Med Qingdao Univ 50: 283-288, 2014.

37. Zhou T, Zu G, Zhang X, Wang X, Li S, Gong X, Liang Z and Zhao J: Neuroprotective effects of ginsenoside Rg1 through the Wnt/ $\beta$-catenin signaling pathway in both in vivo and in vitro models of Parkinson's disease. Neuropharmacology 101: 480-489, 2016.

38. Zhou YC, Chen XC, Zhu YG, Fang F and Chen LM: Down-regulation of oxidative stress is the possible mechanism of ginsenoside $\mathrm{Rg} 1$ protecting the substantia nigra neurons in PD mice. Chin J Clin Pharmacol Ther 8, 2003 (In Chinese).

39. Zhu FX, Chang HM, Duan Y, Li PY and Wang SX: Effect of ginsenoside Rg1 on the expressions of tyrosine hydroxylase,ephrin B2 and phosphorylated c-Jun in substantia nigra of mice with Parkinson's disease. J Xinxiang Med Univ 31: 781-785, 2014.

40. Fleming SM, Salcedo J, Fernagut PO, Rockenstein E, Masliah E, Levine MS and Chesselet MF: Early and progressive sensorimotor anomalies in mice overexpressing wild-type human alpha-synuclein. J Neurosci 24: 9434-9440, 2004.

41. Macleod MR, O'Collins T, Howells DW and Donnan GA Pooling of animal experimental data reveals influence of study design and publication bias. Stroke 35: 1203-1208, 2004.

42. Moher D, Pham B, Jones A, Cook DJ, Jadad AR, Moher M, Tugwell $\mathrm{P}$ and Klassen TP: Does quality of reports of randomised trials affect estimates of intervention efficacy reported in meta-analyses? Lancet 352: 609-613, 1998.

43. Bebarta V, Luyten D and Heard K: Emergency medicine animal research: Does use of randomization and blinding affect the results? Acad Emerg Med 10: 684-687, 2003.

44. Bowenkamp KE, David D, Lapchak PL, Henry MA, Granholm AC, Hoffer BJ and Mahalik TJ: 6-hydroxydopamine induces the loss of the dopaminergic phenotype in substantia nigra neurons of the rat. A possible mechanism for restoration of the nigrostriatal circuit mediated by glial cell line-derived neurotrophic factor. Exp Brain Res 111: 1-7, 1996.
45. Ara J, Przedborski S, Naini AB, Jackson-Lewis V, Trifiletti RR, Horwitz $J$ and Ischiropoulos $\mathrm{H}$ : Inactivation of tyrosine hydroxylase by nitration following exposure to peroxynitrite and 1-methyl-4-phenyl-1,2,3,6-tetrahydropyridine (MPTP). Proc Natl Acad Sci USA 95: 7659-7663, 1998.

46. Kuhn DM, Aretha CW and Geddes TJ: Peroxynitrite inactivation of tyrosine hydroxylase: Mediation by sulfhydryl oxidation, not tyrosine nitration. J Neurosci 19: 10289-10294, 1999.

47. Blanchard-Fillion B, Souza JM, Friel T, Jiang GC, Vrana K, Sharov V, Barrón L, Schöneich C, Quijano C, Alvarez B, et al: Nitration and inactivation of tyrosine hydroxylase by peroxynitrite. J Biol Chem 276: 46017-46023, 2001.

48. Jackson-Lewis V and Przedborski S: Protocol for the MPTP mouse model of Parkinson's disease. Nat Protoc 2: 141-151, 2007.

49. Zhao Q, Yang M, Deng Y, Yu H, Wang L, Teng F, Cho K, Ma H, Wu P, Li X, et al: The safety evaluation of salvianolic acid B and ginsenoside Rg1 combination on mice. Int J Mol Sci 16: 29345-29356, 2015.

50. Zhang ZL, Fan Y and Liu ML: Ginsenoside Rg1 inhibits autophagy in H9c2 cardiomyocytes exposed to hypoxia/reoxygenation. Mol Cell Biochem 365: 243-250, 2012.

51. Lu D, Zhu LH, Shu XM, Zhang CJ, Zhao JY, Qi RB, Wang HD and Lu DX: Ginsenoside Rg1 relieves tert-Butyl hydroperoxide-induced cell impairment in mouse microglial BV2 cells. J Asian Nat Prod Res 17: 930-945, 2015.

52. Li SS, Ye JM, Deng ZY, Yu LX, Gu XX and Liu QF: Ginsenoside-Rg1 inhibits endoplasmic reticulum stress-induced apoptosis after unilateral ureteral obstruction in rats. Ren Fail 37: 890-895, 2015.

53. Liu Y, Yi L, Wang L, Chen L, Chen X and Wang Y: Ginsenoside Rg1 protects human umbilical cord blood-derived stromal cells against tert-Butyl hydroperoxide-induced apoptosis through Akt-FoxO3a-Bim signaling pathway. Mol Cell Biochem 421: 75-87, 2016.

54. Huo DS, Zhang M, Cai ZP, Dong CX, Wang H and Yang ZJ: The role of nerve growth factor in ginsenoside Rg1-induced regeneration of injured rat sciatic nerve. J Toxicol Environ Health A 78: $1328-1337,2015$

55. Miao HH, Zhen Y, Ding GN, Hong FX, Xie ZC and Tian M: Ginsenoside $\operatorname{Rg} 1$ attenuates isoflurane-induced caspase-3 activation via inhibiting mitochondrial dysfunction. Biomed Environ Sci 28: 116-126, 2015

56. Liao B, Newmark $\mathrm{H}$ and Zhou R: Neuroprotective effects of ginseng total saponin and ginsenosides Rb1 and Rg1 on spinal cord neurons in vitro. Exp Neurol 173: 224-234, 2002.

57. Zhang YF, Fan XJ, Li X, Peng LL, Wang GH, Ke KF and Jiang ZL: Ginsenoside Rg1 protects neurons from hypoxic-ischemic injury possibly by inhibiting $\mathrm{Ca}^{2+}$ influx through NMDA receptors and L-type voltage-dependent $\mathrm{Ca}^{2+}$ channels. Eur J Pharmacol 586: 90-99, 2008

58. Liu Z, Qi Y, Cheng Z, Zhu X, Fan C and Yu SY: The effects of ginsenoside $\mathrm{Rg} 1$ on chronic stress induced depression-like behaviors, BDNF expression and the phosphorylation of PKA and CREB in rats. Neuroscience 322: 358-369, 2016.

This work is licensed under a Creative Commons Attribution-NonCommercial-NoDerivatives 4.0 International (CC BY-NC-ND 4.0) License. 